\title{
One-loop divergences of quantized Einstein-Maxwell fields*
}

\author{
S. Deser and P. van Nieuwenhuizen \\ Department of Physics, Brandeis University, Waltham, Massachusetts 02154 \\ (Received 8 April 1974)
}

\begin{abstract}
All one-loop divergences of the quantized Einstein-Maxwell system are calculated, using dimensional regularization and the background-field method. The resulting counterterms, including photon and graviton ghost contributions, are quadratic in curvatures when evaluated on the mass shell, and cannot be renormalized away by rescaling. Brans-Dicke theory is also nonrenormalizable; source-free Einstein theory with cosmological term is (formally) renormalizable, as are Weyl models.
\end{abstract}

\section{INTRODUCTION}

Quantization of general relativity has been the subject of much investigation. While canonical analysis of the gravitational field has established its dynamical modes and Hamiltonian, as well as the constraints and gauge components, this method has had limited success to date in calculating $S$ matrix elements, owing to problems of ordering of noncommuting operators and the practical disadvantages of calculating with noncovariant Feynman rules.

Work by Feynman, ${ }^{1}$ DeWitt, ${ }^{2}$ and others on explicitly (Lorentz) covariant quantization has led to understanding of the classical and essentially quantum effects as corresponding to tree and closedloop diagrams, using for example, the backgroundfield method ${ }^{2}$ to separate (virtual) loop lines from the remaining external (virtual or real) particle lines. Further clarification of the accompanying ghost mechanism for restoring unitarity in closedloop diagrams of non-Abelian gauge theories came with the work of Faddeev and Popov ${ }^{3}$ on covariant quantization. Equivalence of canonical and covariant quantization has also been demonstrated for vector gauge theories. ${ }^{3}$

The tree diagrams contain the classical aspects of the theory, and the field generated by the sum of all tree graphs satisfies the (classical) Einstein equations. However, an assessment of closed-loop effects in general relativity, especially the type of divergences which they generate, has been lacking until recently. Thus, one may find in the literature opinions that general relativity will act as a universal cutoff for all divergences (including its own), as well as the opposite view that it is violently nonrenormalizable owing to the dimensionality of the gravitational coupling constant. ${ }^{4}$ It may be that both viewpoints are correct and that general relativity is highly divergent in perturbation expansion, but will resolve all cutoff problems when treated with appropriate future nonperturba- tive methods. Or, it may be necessary to modify the theory through nonminimal interactions. In any case, it is essential first to understand the nature of the divergences of conventional theory.

Quite recently, the divergence question received its first complete treatment, at the one-loop level, in the work of 't Hooft and Veltman. ${ }^{5}$ Using dimensional regularization, ${ }^{6}$ they derived a general algorithm for obtaining the divergent contributions, for $r$ ather general systems, arising from one closed loop with arbitrary numbers of attached external lines. These contributions were expressed in terms of a gauge-independent counter-Lagrangian depending on the background fields (external lines). One-loop renormalizability could then be determined by evaluating the counterterms on the mass shell (i.e., by inserting the background field's classical field equations). The $S$ matrix is renormalizable if these counterterms then vanish or if they are proportional to terms in the original Lagrangian (the usual renormalization through rescaling). The third possibility is that the counterterms are of a new form (as for example in $\phi^{4}$ theory); if, to all loop orders, only a finite number of such terms are found, the theory is still renormalizable. Reference 5 specifically treated the source-free Einstein field and coupled Einstein plus scalar fields. The free field turned out to be one-loop finite, but this is due to a peculiar identity in four-dimensional Riemann geometry which reduces an a priori unrenormalizable term to a combination which vanishes on the mass shell [Eq. (4.7)]. In the presence of a scalar field, however, renormalizability was lost.

Since elementary scalar fields may not exist, however, it is important to discover whether coupling to more physical systems such as photons and fermions also exhibits the same difficulties. We report here the results for the coupled Maxwell-Einstein system: It is nonrenormalizable. So is Brans-Dicke theory, even in the absence of matter. For completeness, we have also con- 
sidered the source-free Einstein theory with cosmological term; although not very physical as a quantum system, it is formally renormalizable. This also seems to be the case for source-free models of the Weyl type. In the accompanying paper, we will deal with fermions, which require separate treatment because vierbein fields, rather than just the metric, must be used there.

\section{BACKGROUND FIELDS AND GAUGE PROPERTIES}

In this section, we set the stage for quantization of the Einstein-Maxwell system. For one-loop purposes, we need only consider vertices with two internal field lines, but arbitrarily many external ones. The background-field method is an appropriate tool; the action is expanded to second order in the quantum fields about an arbitrary classical background field, which represents all possible tree graphs attached to the loop. Since only the quantized fields are treated dynamically, it is useful to recast the gauge invariances of the total fields in terms of those of the quantum fields alone. These gauge invariances will be duly broken, as usual, by a choice of quantum gauge, which in the covariant quantization approach entails unitarity-restoring ghost-loop diagrams. The final dependence on the background fields will of course be gauge-invariant, since no gauge choices are made for them, and manifest covariance is kept throughout.

We start with the usual Maxwell-Einstein Lagrangian,

$$
\begin{aligned}
\mathfrak{L} & \equiv \mathscr{L}(\bar{g}, \bar{F}) \\
& =-(-\bar{g})^{1 / 2}\left[\kappa^{-2} R(\bar{g})+\frac{1}{4} \bar{F}_{\mu \nu} \bar{F}_{\alpha \beta} \bar{g}^{\mu \alpha} \bar{g}^{\nu \beta}\right],
\end{aligned}
$$

where $R(\bar{g})=\bar{g}^{\alpha \beta} R_{\alpha \beta}(\bar{g})=\bar{g}^{\alpha \beta} R_{\alpha \beta \mu}^{\mu}$ is the scalar curvature, with sign convention given by $R_{\alpha \beta \nu}^{\mu} \sim$ $+\partial_{\beta} \Gamma_{\alpha \nu}^{\mu}, \bar{F}_{\mu \nu} \equiv \partial_{\mu} \bar{A}_{\nu}-\partial_{\nu} \bar{A}_{\mu}$, and $\kappa$ is related to Newton's constant by $\kappa^{2}=16 \pi \gamma$; the metric signature is +2 and $\hbar=c=1$, so that $\kappa$ has the dimensions of a length.

The fields $\bar{g}_{\mu \nu}$ and $\bar{A}_{\alpha}$ are now written as sums of background fields $(g, A)$ and quantum fields $(h, a)$ according to?

$$
\bar{g}_{\mu \nu}=g_{\mu \nu}+\kappa h_{\mu \nu}, \quad \bar{A}_{\alpha}=\kappa^{-1} A_{\alpha}+a_{\alpha},
$$

and of course all functions of $\bar{g}_{\mu \nu}$, such as its inverse $\bar{g}^{\mu \nu}$, have their expansion defined by that of $\bar{g}_{\mu \nu}$ :

$$
\begin{aligned}
& \bar{g}^{\mu \nu}=g^{\mu \nu}-\kappa h^{\mu \nu}+\kappa^{2} h^{\mu}{ }_{\alpha} h^{\alpha \nu}+\cdots, \\
& (-\bar{g})^{1 / 2}=(-g)^{1 / 2}\left[1+\frac{1}{2} \kappa h\right. \\
& \left.-\frac{1}{4} \kappa^{2}\left(h^{\mu \nu} h_{\mu \nu}-\frac{1}{2} h^{2}\right)+\cdots\right] .
\end{aligned}
$$

All tensor operations, such as moving indices and covariant differentiation $\left(D_{\alpha}\right)$, are performed with respect to the background metric $g_{\mu \nu}$ (e.g., $h \equiv h_{\alpha}^{\alpha}$ $\left.\equiv g^{\alpha \beta} h_{\alpha \beta}\right)$. We have included a coefficient $\kappa$ in our definition of $h_{\mu \nu}$; this is required so that $h_{\mu \nu}$ have dimensions of a Bose field, and the unit numerical coefficient ensures correct canonical form of Eq. (2.1) for $h_{\mu \nu}$ as a spin-2 field in the background space. It is also convenient, for future purposes, to define the background vector potentials $A_{\mu}$ to be dimensionless by including a $\kappa^{-1}$ factor there. It is already apparent from (2.2b) that the coefficients of $h_{\alpha \beta} h_{\mu \nu}$ will be highly nonlinear in the background metric, corresponding to arbitrarily many external gravitons emerging from each vertex in the loop diagrams. The expansion of the action (2.1) in quantum fields is given by

$$
\begin{aligned}
\int d x \mathscr{L}\left(g+\kappa h, \kappa^{-1} A+a\right)= & \kappa^{-2} \int \mathcal{L}(g, A)+\kappa^{-1} \int \mathscr{L}_{1} \\
& +\int \mathscr{L}_{2}+\kappa \int \mathscr{L}_{3}+\cdots
\end{aligned}
$$

Note that expansion in quantum fields is also one in powers of $\kappa$. The $\kappa^{-2}$ part is the classical action, $\mathscr{L}_{1}$ is linear in the quantum fields, and $\mathscr{L}_{2}$ is the quadratic term of interest. Since the coefficients of $(h, a)$ in $\mathscr{L}_{1}$ are just the variational derivatives of $\mathscr{L}$ with respect to the classical fields, they will vanish when $(g, A)$ satisfy the Maxwell-Einstein equations

$$
\begin{aligned}
G_{\mu \nu} & =-\frac{1}{2} T_{\mu \nu}, \quad D_{\alpha} F^{\alpha \beta}=0=D_{\alpha} F_{\beta \gamma}+\mathrm{cycl} ., \\
G_{\mu \nu} & \equiv R_{\mu \nu}-\frac{1}{2} g_{\mu \nu} R, \\
T_{\mu \nu} & \equiv F_{\mu \alpha} F_{\nu}^{\alpha}-\frac{1}{4} g_{\mu \nu} F^{\alpha \beta} F_{\alpha \beta} .
\end{aligned}
$$

Thus the background fields represent all possible tree graphs. We note for later use some algebraic consequences of the field equations,

$$
\begin{aligned}
R=\frac{1}{2} T_{\alpha}^{\alpha} & =0, \\
4 R_{\alpha \beta} R^{\alpha \beta} & =T_{\alpha \beta} T^{\alpha \beta} \\
& =\operatorname{tr}\left(F^{4}\right)-\frac{1}{4}\left(\operatorname{tr} F^{2}\right)^{2}, \\
R_{\alpha \beta} T^{\alpha \beta}= & -2 R_{\alpha \beta} R^{\alpha \beta} .
\end{aligned}
$$

The quadratic part $\mathscr{L}_{2}$ has the form

$$
\begin{aligned}
\int d x \mathscr{L}_{2}=\int d x(-g)^{1 / 2}[ & -\frac{1}{2}\left(D_{\nu} h_{\alpha \beta}\right) P^{\alpha \beta \rho \sigma}\left(D^{\nu} h_{\rho \sigma}\right)+\frac{1}{2}\left(h_{\mu}-\frac{1}{2} D_{\mu} h\right)^{2}-\frac{1}{2}\left(D_{\nu} a_{\mu}\right)^{2}+\frac{1}{2}\left(D_{\mu} a_{\nu}\right)\left(D^{\nu} a^{\mu}\right) \\
& \left.+\frac{1}{2} h_{\alpha \beta}\left(X_{g}+X_{e}\right)^{\alpha \beta \rho \sigma} h_{\rho \sigma}+h_{\alpha \beta} Q^{\alpha \beta \rho \sigma} D_{\rho} a_{\sigma}\right],
\end{aligned}
$$


where $h_{\mu} \equiv D^{\nu} h_{\mu \nu}$. We have divided the action into diagonal kinetic parts of the form $\phi \square \phi$, remaining kinetic terms (gauge parts) soon to be removed by appropriate gauge-breaking terms, a mixing terms bilinear in $h$ and $f$, and a nonderivative term in $h h$ (there are clearly no $h D h$ terms by dimensions). All bilinear terms in $h$, except for $X_{e}$, come from expansion of the scalar curvature, while $X_{e}$ and the remaining terms come from the Maxwell part. Note that we have chosen to write $f_{\mu \nu} \equiv \partial_{\mu} a_{\nu}-\partial_{\nu} a_{\mu}$ in its equivalent covariant version $D_{\mu} a_{\nu}-D_{\nu} a_{\mu}$. We now specify the remaining symbols which are computed straightforwardly from the expansion (cf. Ref. 5 for the purely gravitational parts):

$$
\begin{aligned}
P^{((\alpha \beta)(\rho \sigma))=} & \frac{1}{2} g^{\alpha \rho} g^{\beta \sigma}-\frac{1}{4} g^{\alpha \beta} g^{\rho \sigma}, \\
X_{g}^{((\alpha \beta)(\rho \sigma))}= & P^{\alpha \beta \rho \sigma} R-g^{\alpha \rho} R^{\beta \sigma}+g^{\alpha \beta} R \\
X_{e}^{((\alpha \beta)(\rho \sigma))=} & P^{\alpha \beta \rho \sigma \frac{1}{4}} F^{2}-\frac{1}{2} F^{\alpha \rho} F^{\beta \sigma} \\
& -g^{\alpha \rho} F_{2}^{\beta \sigma}+\frac{1}{2} g^{\alpha \beta} F_{2}^{\rho \sigma}, \\
Q^{(\alpha \beta)[\rho \sigma]}= & 2 g^{\alpha \rho} F^{\beta \sigma}-\frac{1}{2} g^{\alpha \beta} F^{\rho \sigma},
\end{aligned}
$$$$
X_{g}^{((\alpha \beta)(\rho \sigma))}=P^{\alpha \beta \rho \sigma} R-g^{\alpha \rho} R^{\beta \sigma}+g^{\alpha \beta} R^{\rho \sigma}+R^{\alpha \rho \beta \sigma} \text {, }
$$

with the definition $F_{2}^{\mu \nu} \equiv F_{2}^{\nu \mu} \equiv F_{\alpha}^{\mu} F^{\nu \alpha}, F_{2 \mu}^{\mu} \equiv F^{2}$. Parentheses (square brackets) around index pairs denote symmetrization (antisymmetrization), while parentheses around four indices means symmetrization also under pair interchange. These symmetries in (2.7) are automatically enforced by the symmetries of the quantum fields multiplying these quantities. We write out one specific example:

$$
P^{\alpha \beta \rho \sigma}=\frac{1}{4}\left(g^{\alpha \rho} g^{\beta \sigma}+g^{\alpha \sigma} g^{\beta \rho}-g^{\alpha \beta} g^{\rho \sigma}\right) .
$$

The propagators of the $h$ and $a$ fields are, in principle, obtained by inverting the coefficients of the kinetic terms in (2.6). However, the gauge invariances of the action preclude inversion until a gauge choice is made. The original action $\overline{\mathfrak{L}}$ was invariant under the finite coordinate and electromagnetic (em) gauges

$$
\begin{aligned}
& \bar{g}_{\mu \nu}^{\prime}\left(x^{\prime}\right)=\bar{g}_{\alpha \beta}(x)\left(\frac{\partial x^{\alpha}}{\partial x^{\prime \mu}}\right)\left(\frac{\partial x^{\beta}}{\partial x^{\prime \nu}}\right), \\
& \bar{A}_{\mu}^{\prime}\left(x^{\prime}\right)=\bar{A}_{\alpha}(x)\left(\frac{\partial x^{\alpha}}{\partial x^{\prime \mu}}\right)+\partial_{\mu} \Lambda,
\end{aligned}
$$

with

$$
\int d^{4} x^{\prime} \mathcal{L}\left(\bar{g}^{\prime}, \bar{A}^{\prime}\right)=\int d^{4} x \mathcal{L}(\bar{g}, \bar{A}) .
$$

Let us recast the infinitesimal equivalents of (2.8) as gauge transformations on the quantum fields alone,

$$
\begin{aligned}
h_{\mu \nu}^{\prime}(x)= & h_{\mu \nu}(x)+\left(g_{\mu \alpha}+\kappa h_{\mu \alpha}\right) \eta^{\alpha}{ }_{, \nu} \\
& +\left(g_{\alpha \nu}+\kappa h_{\alpha \nu}\right) \eta^{\alpha}{ }_{, \mu}+\eta^{\alpha}\left(g_{\mu \nu}+\kappa h_{\mu \nu}\right)_{, \alpha}, \\
a_{\mu}^{\prime}(x)= & a_{\mu}(x)+\left(A_{\alpha}+\kappa a_{\alpha}\right) \eta^{\alpha}{ }_{, \mu} \\
& +\eta^{\alpha}\left(A_{\mu}+\kappa a_{\mu}\right)_{, \alpha}+\partial_{\mu} \Lambda,
\end{aligned}
$$

where $x^{\alpha}-x^{\prime \alpha}=\kappa \eta^{\alpha}$ and the subscript comma denotes ordinary differentiation. We emphasize that this is merely a rewriting of (2.8), but with the background fields left untransformed; their invariances will be manifest at the end. The transport terms, proportional to nondifferentiated $\eta^{\alpha}$, combine with the ordinary derivatives in the usual way to yield covariant derivatives $D_{\alpha}$ with respect to $g_{\mu \nu}$, and we have

$$
\begin{aligned}
h_{\mu \nu}^{\prime}(x)= & h_{\mu \nu}(x)+\left(g_{\mu \alpha} D_{\nu}+g_{\nu \alpha} D_{\mu}\right) \eta^{\alpha} \\
& +\kappa\left[\left(h_{\mu \alpha} D_{\nu}+h_{\nu \alpha} D_{\mu}\right) \eta^{\alpha}+\eta^{\alpha} D_{\alpha} h^{\mu \nu}\right],
\end{aligned}
$$

while

$$
\begin{aligned}
a_{\mu}^{\prime}(x)= & a_{\mu}(x)+\left(A_{\alpha} D_{\mu} \eta^{\alpha}+\eta^{\alpha} D_{\alpha} A_{\mu}\right)+D_{\mu} \Lambda \\
& +\kappa\left(a_{\alpha} D_{\mu} \eta^{\alpha}+\eta^{\alpha} D_{\alpha} a_{\mu \nu}\right) .
\end{aligned}
$$

The $\eta$ transformation contains both homogeneous and inhomogeneous terms. The latter involve $A_{\mu}$ rather than the field $F_{\mu \nu}$; while this is actually harmless, we may restore manifest electromagnetic gauge invariance of the external field by taking advantage of the freedom of $\Lambda$ transformations ${ }^{8}$ to go to a new basis

$$
\Lambda=-\eta^{\alpha} A_{\alpha}+\eta^{5},
$$

in which $a_{\mu}$ transforms as

$a_{\mu}^{\prime}=a_{\mu}+\eta^{\alpha} F_{\alpha \mu}+D_{\mu} \eta^{5}+\kappa\left(a_{\alpha} D_{\mu} \eta^{\alpha}+\eta^{\alpha} D_{\alpha} a_{\mu}\right)$

We shall return to this when deriving the ghost Lagrangians. The infinitesimal transformations (2.10) leave the complete action $\overline{\mathscr{L}}$ invariant. Whenever there is a gauge invariance, one must, in the convariant quantization scheme, supply an appropriate gauge-breaking term to avoid counting different gauge forms of the same state separately. If one also wants to be able to define propagators for the gauge fields, then these gauge-breaking terms must cancel those "bad" second-derivative parts of the original action which we have called "gauge parts." In our case, this could be accomplished by adding any term whose leading derivative behavior is $-\frac{1}{2}\left(\partial^{\alpha} h_{\mu \alpha}-\partial_{\mu} h\right)\left(\partial^{\beta} h_{\nu \beta}-\partial_{\nu} h\right) g^{\mu \nu}$. The simplest and most useful, because it keeps manifest covariance in the background metric, is the combination $-\frac{1}{2}\left(h_{\mu}-D_{\mu} h\right)^{2}$ in terms of covariant derivatives, which cancels the gauge parts of (2.6). The unitarity-restoring ghost terms which must be added to $\overline{\mathscr{L}}$ are then obtained from the particular choice of gauge-breaking terms. ${ }^{9}$ 


\section{QUANTIZATION, GAUGES, AND GHOSTS}

We perform covariant quantization in combined deDonder (harmonic) and Lorentz gauges, which we impose by adding gauge-breaking terms to $\mathscr{L}_{2}$. It will then be possible to define propagators for the photons and gravitons; in turn, gauge breaking will induce five Faddeev-Popov ghosts: a gravitational vector and an electromagnetic scalar. The quantum Lagrangian will then be the sum of a nonghost (3.3) and a ghost (3.12) part.
As suggested by (2.6), appropriate gauge-breaking terms are

$$
\begin{aligned}
& \mathscr{L}_{\mathrm{EB}}=-\frac{1}{2}(-g)^{1 / 2}\left(h_{\mu}-D_{\mu} h\right)^{2}, \\
& \mathscr{L}_{\mathrm{MB}}=-\frac{1}{2}(-g)^{1 / 2}\left(D_{\mu} a^{\mu}\right)^{2} .
\end{aligned}
$$

Use of the Ricci identity

$$
\begin{aligned}
& \left(D_{\alpha} D_{B}-D_{\beta} D_{\alpha}\right) V^{\mu}=R_{\gamma \alpha \beta}^{\mu} V^{\gamma}, \\
& \left(D_{\mu} D_{B}-D_{B} D_{\mu}\right) V^{\mu}=-R_{\mu \beta} V^{\mu}
\end{aligned}
$$

gives for the nonghost Lagrangian (2.6) plus (3.1)

$$
\mathscr{L}_{2}+\mathscr{L}_{\mathrm{EB}}+\mathscr{L}_{\mathrm{MB}}=(-g)^{1 / 2}\left[-\frac{1}{2}\left(D_{\nu} h\right) P\left(D^{\nu} h\right)-\frac{1}{2}\left(D_{\mu} a_{\nu}\right)^{2}+\frac{1}{2} a_{\alpha} R^{\alpha \beta} a_{B}+\frac{1}{2} h\left(X_{g}+X_{e}\right) h+h_{\alpha \beta} Q^{\alpha \beta \rho \sigma} D_{\rho} a_{\sigma}\right],
$$

omitting obvious indices. To simplify the propagators, we diagonalize the two kinetic terms by the following procedure. ${ }^{5}$ First introduce complex fields by adding identical actions in terms of fields $h_{1}, h_{2}, a_{1}, a_{2}$ and call

$$
h \equiv\left(h_{1}+i h_{2}\right) 2^{-1 / 2}, \quad a \equiv\left(a_{1}+i a_{2}\right) 2^{-1 / 2} .
$$

One may verify the identity

$$
\sum_{i=1}^{2} h_{i} Q D a_{i}=h^{*} Q D a-a^{*} Q D h-a^{*}(D Q) h .
$$

Now we may absorb the explicit factors of $P$ into $h_{\mu \nu}^{*}$ and $g_{\rho \sigma}$ into $a_{\alpha}^{*}$ by setting $h_{\alpha \beta}^{*} P^{\alpha \beta \rho \sigma} \rightarrow h_{\rho \sigma}^{*}$ and $a_{\alpha}^{*} g^{\alpha \beta} \rightarrow a_{B}^{*}$; this is just a standard field redefinition which will not alter physical results. A more complicated problem is that the covariant derivatives in, say, $h_{\alpha \beta}^{*} D_{\nu} D^{\nu} h_{\alpha \beta}$ act also on the tensor indices of $h$ and thus connect different components. This is not the case, however, for the leading second-derivative parts of $D^{2}$, and we may therefore define a new derivative $\tilde{D}$ which does not act on explicit field indices $\left(\tilde{D}_{\nu} h_{\alpha \beta} \equiv \partial_{\nu} h_{\alpha \beta}\right)$ at the cost of introducing lower-derivative terms. We quote the result in terms of the "d'Alembertian" $\tilde{D}_{\mu} \tilde{D}^{\mu}$ $\equiv g^{\mu \nu}\left(\partial_{\mu} \partial_{\nu}-\Gamma_{\mu \nu}^{\sigma} \partial_{\sigma}\right):$

$$
\begin{aligned}
h_{\alpha \beta}^{*} D_{\nu} D^{\nu} h_{\alpha \beta}= & h_{\alpha \beta}^{*} \tilde{D}_{\nu} \tilde{D}^{\nu} h_{\alpha \beta}+2 h_{\alpha \beta}^{*} \mathfrak{N}_{\alpha \beta}^{\mu}{ }^{\rho \sigma} \tilde{D}_{\mu} h_{\rho \sigma} \\
& +h_{\alpha \beta}^{*} \mathfrak{T}_{\alpha \beta}^{\rho \sigma} h_{\rho \sigma}, \\
a_{\alpha}^{*} D_{\nu} D^{\nu} a_{\alpha}= & a_{\alpha}^{*} \tilde{D}_{\nu} \tilde{D}^{\nu} a_{\alpha}+2 a_{\alpha}^{*} n_{\alpha}^{\mu \beta} \tilde{D}_{\mu} a_{\beta} \\
& +a_{\alpha}^{*} \tau_{\alpha}^{\beta} a_{\beta},
\end{aligned}
$$

with

$$
\begin{aligned}
& \mathfrak{R}_{(\alpha \beta)}^{\mu(\rho \sigma)}=-2 g^{\mu \tau} \Gamma_{\tau \alpha}^{\rho} \delta_{\beta}^{\sigma}, \quad \mathfrak{T}_{\alpha \beta}^{\rho \sigma}=\left(D_{\mu} \mathfrak{N}^{\mu}+\mathfrak{N}_{\mu} \mathfrak{N}^{\mu}\right)_{\alpha \beta}^{\rho \sigma} \\
& n_{\alpha}^{\mu \beta}=-g^{\mu \tau} \Gamma_{\tau \alpha}^{\rho}, \quad \tau_{\alpha}^{\beta}=\left(D_{\mu} n^{\mu}+n_{\mu} n^{\mu}\right)_{\alpha}^{\beta},
\end{aligned}
$$

and $D_{\mu}$ in (3.8) acts only on the index $\mu$ :

$$
\left(D_{\mu} n^{\mu}\right)_{\alpha}^{\beta} \equiv \partial_{\mu} n_{\alpha}^{\mu \beta}+\Gamma_{\mu \sigma}^{\mu} n_{\alpha}^{\sigma \beta}, \quad n_{\mu} \equiv g_{\mu \nu} n^{\nu} .
$$

With these rearrangements and insertions, the nonghost Lagrangian has now been put into the desired "scalar" form in terms of $10+4$ independent complex fields $\phi_{i}=\{h, a\}$,

$$
\begin{gathered}
\mathcal{L}_{\mathrm{NG}}=(-g)^{1 / 2}\left(\phi_{i}^{*} D_{\nu} D^{\nu} \phi_{i}+2 \phi_{i}^{*} N^{\mu, i j} \partial_{\mu} \phi_{j}\right. \\
\left.+\phi_{i}^{*} M^{i j} \phi_{j}\right),
\end{gathered}
$$

where the $N^{\mu}$ and $M$ are matrices in the $(h, a)$ space:

$$
\begin{aligned}
N_{\mathrm{NG}}^{\mu} & =\left(\begin{array}{cc}
\mathfrak{R}_{\alpha \beta}^{\mu}{ }^{\rho \sigma} & \left(P^{-1} \frac{1}{2} Q^{\mu}\right)_{\alpha \beta}^{\delta} \\
-\frac{1}{2} g_{\gamma \lambda} Q^{\rho \sigma \mu \lambda} & n_{\gamma}^{\mu \delta}
\end{array}\right), \\
M_{\mathrm{NG}} & =\left(\begin{array}{cc}
P^{-1}\left(X_{g}+X_{e}\right)+\mathfrak{T} & 0 \\
-g_{\gamma \lambda} \partial_{\mu} Q^{\rho \sigma \mu \lambda} & R_{\gamma}^{\delta}+\tau_{\gamma}^{\delta}
\end{array}\right) .
\end{aligned}
$$

There remains the ghost contribution, which is to be obtained in the usual way from the gauge-breaking terms (3.1). One subjects the square roots of the latter,

$$
\begin{aligned}
& C_{a}=(-g)^{1 / 4} e_{a}^{\mu}\left(h_{\mu}-\frac{1}{2} D_{\mu} h\right), \\
& C_{5}=(-g)^{1 / 4} D_{\mu} a^{\mu},
\end{aligned}
$$

to the gauge transformations (2.10). Here $e_{a}{ }^{\mu}$ is a vierbein field which represents a square root of the metric $\left(e_{a}{ }^{\mu} e_{b}{ }^{\nu} \eta^{a b}=g^{\mu \nu}\right)$ with respect to the local Minkowski frame $\eta_{a b}$. The ghost Lagrangian is then defined as

$$
\mathscr{L}_{G}=\sum_{a, b=1}^{5} \phi^{a *}\left(\frac{\partial C_{a}}{\partial \eta^{b}}\right) \phi^{b},
$$

where the $\phi^{a}$ are the ghost fields. Since $\mathscr{L}_{G}$ is already bilinear in the quantum operator $s \phi^{*} \phi$, we may drop any dependence on $(h, a)$ in $C_{a}$. Thus, only the inhomogeneous terms of (2.10) contribute: 


$$
\begin{aligned}
\mathscr{L}_{G}= & (-g)^{1 / 4}\left(\phi^{* \alpha}, \chi^{*}\right) \\
& \times\left(\begin{array}{cc}
e_{\alpha \beta} D_{\nu} D^{\nu}-R_{\alpha \beta} & 0 \\
-\left(D^{\lambda} F_{\lambda \beta}\right)-F_{\lambda \beta} D^{\lambda} & D_{\nu} D^{\nu}
\end{array}\right)\left(\begin{array}{c}
\phi^{\beta} \\
\chi
\end{array}\right) .
\end{aligned}
$$

We again absorb factors such as $(-g)^{1 / 4} e_{\alpha \beta}$ in the $\phi^{*}$, and go to $\tilde{D}_{\nu}$ to diagonalize $\mathscr{L}_{G}$ in the desired form (3.9), with

$$
N_{G}^{\mu}=\left(\begin{array}{cc}
n_{\alpha}^{\mu \beta} & 0 \\
-\frac{1}{2} F_{\lambda \beta} & 0
\end{array}\right), \quad M_{G}=\left(\begin{array}{cc}
-R_{\alpha}^{\beta}+\tau_{\alpha}^{\beta} & 0 \\
-D^{\lambda} F_{\lambda}^{\beta} & 0
\end{array}\right) .
$$

Note that the scalar ghost is now equivalent to a normal scalar field (with negative residue) and the vector ghost is almost of the form of a (negative probability) Maxwell field, except that in $M,-R_{\alpha}^{\beta}$ occurs instead of $+R_{\alpha}^{\beta}$.

We note the unexpected contribution of the photon ghost, whose absence in flat-space electrodynamics is usually ascribed to the Abelian nature of the em gauges (but that feature holds here as well). The contribution is due to the fact that the gauge breaking term $C_{5}$ of (3.11) depends on the external metric through $D_{\mu}$. We could of course have stuck to ordinary derivatives on all $a_{\alpha}$, in which case $C_{5}$ would look like $g^{\mu \nu} \partial_{\mu} a_{\nu}$ and there would still be a (different) contribution from it. Note also that there is no vector-scalar ghost mixing because (3.12) contains a $\phi^{*} \phi^{\beta}$ term, but no conjugate to it.

\section{THE COUNTER-LAGRANGIAN}

Having written the action in the scalar form (3.9), we may immediately write down the counter-Lagrangian $\Delta \mathscr{L}(g, F)$ which eliminates all the oneloop divergences in the dimensional-regularization framework. It is given by the algorithm of Ref. 5 , in terms of the dimensional-regularization cutoff $(1 / \epsilon)$, as

$$
\begin{aligned}
\Delta \mathscr{L}=\frac{1}{\epsilon}(-g)^{1 / 2}\{\operatorname{tr} & {\left[\frac{1}{12} Y_{\mu \nu} Y^{\mu \nu}+\frac{1}{4} X^{2}\right.} \\
& \left.\left.+\frac{1}{60}\left(R_{\mu \nu} R^{\mu \nu}-\frac{1}{3} R^{2}\right)\right]\right\},
\end{aligned}
$$

where $Y_{\mu \nu}$ and $X$ are determined from (3.10), (3.13) according to

$$
\begin{aligned}
& Y_{\mu \nu}=\partial_{\mu} N_{\nu}-\partial_{\nu} N_{\mu}+N_{\mu} N_{\nu}-N_{\nu} N_{\mu}, \\
& X=M-D_{\mu} N^{\mu}-N_{\mu} N^{\mu}-\frac{1}{6} R .
\end{aligned}
$$

Here the $D^{\prime}$ 's act only on the single index shown, as in (3.8c). The trace is to be taken over the $10\left(h_{\mu \nu}\right)+4\left(a_{\mu}\right)+4\left(\phi^{\alpha}\right)+1(\phi)$ independent components, but the nonghost part must be divided by two in order to undo the doubling into complex fields, while the ghost part must, as usual, acquire an extra minus sign.

Before giving explicit results, which involve rather lengthy calculations, one can say a good deal about the type of counterterms to be expected, making use of dimensionality and the gauge covariance in the background fields. Although the general ring graph has an arbitrary number of external lines, and therefore may have arbitrary powers of $R_{\mu \nu \alpha \beta}$ or $F_{\mu \nu}$, the divergent part in which the loop shrinks to a point does not. This is the case even though ring graphs with arbitrary numbers of external gravitons have the same degree of divergence, and is due to the fact that dimensional regularization employs a dimensionless (logarithmic) cutoff $(1 / \epsilon)$. Thus (with our units) $\Delta \&$, which has dimensions $L^{-4}$, can only involve a small number of building blocks involving the covariant quantities $R_{\nu \alpha \beta}^{\mu}\left(\sim L^{-2}\right), F_{\mu \nu} F_{\alpha \beta}$ $\left(\sim L^{-2}\right.$ ) (since there must be an even number of external photon lines), and of course the metric itself. The dimensional coupling constant $\kappa$ has disappeared from $\mathscr{L}_{1}$ and so cannot enter the $\Delta \mathscr{L}$. Covariant derivatives $D_{\alpha}$ may also appear, but (since all other building blocks have an even number of indices) only in the bilinear combination $D_{\alpha} D_{\beta}$. Thus, only expressions bilinear in products of $R_{\nu \alpha \beta}^{\mu}, F_{\mu \nu} F_{\alpha \beta}$, and $D_{\alpha} D_{\beta}$ are permitted. ${ }^{10}$ Since we are not interested in total divergences, $D D$ can only occur in the form $(D F)(D F)$, with various index contractions. But such terms can be recast, using the Ricci identities and the cyclic identity $F_{[\mu \nu ; \alpha]}=0$, into nonderivative form plus the basic combination $\left(D_{\mu} F^{\mu \nu}\right)^{2}$. For example,

$$
\begin{aligned}
\left(D_{\alpha} F_{\beta \gamma}\right)\left(D^{\alpha} F^{\beta \gamma}\right)= & 2 R_{\alpha \beta} F_{2}^{\alpha \beta}+R_{\alpha \beta \gamma \delta} F^{\alpha \beta} F^{\gamma \delta} \\
& +2\left(D_{\beta} F^{\beta \gamma}\right)\left(D^{\alpha} F_{\alpha \gamma}\right) \\
\left(D_{\alpha} F_{B \gamma}\right)\left(D^{\beta} F^{\alpha \gamma}\right)= & R_{\alpha \beta} F_{2}^{\alpha \beta}+R_{\alpha \beta \gamma \delta} F^{\alpha \gamma} F^{\beta \delta} \\
& +\left(D_{B} F^{\beta \gamma}\right)\left(D^{\alpha} F_{\alpha \gamma}\right) \\
= & \frac{1}{2}\left(D_{\alpha} F_{\beta \gamma}\right)^{2} .
\end{aligned}
$$

Thus we are left with $\left(D_{\alpha} F^{\alpha \gamma}\right)^{2}$ and terms bilinear in the curvature and $F F$. There cannot be candidates involving a single $\epsilon^{\alpha \beta \gamma \delta}$ symbol by parity (actually, terms like $R \in R$ are total divergences anyway ${ }^{11}$ ), while two $\epsilon$ 's can always be expressed in terms of $g_{\mu \nu}$ 's. Also the cyclic identity $R_{\mu[\nu \alpha \beta]}$ $=0$ enables us to rewrite, e.g.,

$$
\begin{aligned}
& R_{\mu \nu \alpha \beta} R^{\mu \alpha \nu \beta}=\frac{1}{2} R_{\mu \nu \alpha \beta} R^{\mu \nu \alpha \beta}, \\
& R_{\mu \nu \alpha \beta} F^{\mu \alpha} F^{\nu \beta}=\frac{1}{2} R_{\mu \nu \alpha \beta} F^{\mu \nu} F^{\alpha \beta} .
\end{aligned}
$$

Thus, the most general $\Delta \mathscr{L}$ has the a priori form 


$$
\begin{aligned}
\Delta \mathcal{L}=(1 / \epsilon)(-g)^{1 / 2} & {\left[c_{1} R_{\mu \nu}{ }^{2}+c_{2} R^{2}+c_{3} R_{\mu \nu \alpha \beta}{ }^{2}+c_{4} T_{\mu \nu}{ }^{2}+c_{5}\left(F^{2}\right)^{2}+c_{6} R_{\mu \nu \alpha \beta} F^{\mu \nu} F^{\alpha \beta}+c_{7} R_{\mu \nu} T^{\mu \nu}\right.} \\
& \left.+c_{8} R F^{2}+c_{9}\left(D_{\mu} F^{\mu \nu}\right)^{2}\right]
\end{aligned}
$$

where we have chosen $\left(T^{\mu \nu}, g^{\mu \nu} F^{2}\right)$ rather than $\left(F_{2}^{\mu \nu}, g^{\mu \nu} F^{2}\right)$ as a basis [see Eq. (2.4)].

Note first that, since $\kappa$ is absent and the cutoff is dimensionless, not only are higher-power terms excluded, but there are also no tadpole contributions and neither $(1 / \epsilon)(-g)^{1 / 2} R$ nor $(1 / \epsilon)(-g)^{1 / 2}$ terms appear, in contrast to the usual momentumcutoff procedure. ${ }^{2}$ This absence of gravitational constant or cosmological constant renormalization is independent of loop order, in contrast to terms $\sim \kappa^{2} R^{3}$, etc., which might arise at the two-loop level. Next, we note that the one-loop renormalizability of source-free Einstein theory can be decided on without any calculation at all. The generic form (4.6) degenerates here into the first three terms $c_{1}, c_{2}, c_{3}$; of these, the first two are renormalizable by the $S$-matrix criterion of renormalizability, ${ }^{12}$ that $\Delta \mathscr{L}$ vanish on shell, ${ }^{5}$ where the background fields obey the classical field equations, i.e., $\left.\Delta \mathscr{L}\right|_{\mathscr{L}_{1}=0}=0$. Thus, renormalizability would hinge on the unlikely cancellation of all $c_{3}$ contributions. However, the situation is saved by an altogether different fact, namely, the identity valid only in four-dimensional Riemannian geometry, that

$$
(-g)^{1 / 2}\left[R_{\mu \nu \alpha \beta}{ }^{2}-4 R_{\mu \nu}^{2}+R^{2}\right]=\text { divergence }
$$

It was first discovered, in the context of Weyl's 1918 scale-invariant theory of gravitation, by $\mathrm{Bach}^{11}$ in 1921. This is an example of a general identity holding in an even number of dimensions, and obtained by saturating appropriate powers of the curvature with $\epsilon$ symbols. ${ }^{13}$ Another relevant relation, referred to earlier, is

$$
R_{\mu \nu \alpha \beta} \epsilon^{\alpha \beta \rho \sigma} R_{\rho \sigma}^{\mu \nu}=\text { divergence, }
$$

which excludes parity-violating counterterms (it corresponds to $F_{\mu \nu} \epsilon^{\mu \nu \alpha \beta} F_{\alpha \beta} \sim \overrightarrow{\mathrm{E}} \cdot \overrightarrow{\mathrm{B}}=$ divergence in electrodynamics). In view of (4.7) and (4.8), we see that source-free Einstein theory is one-loop renormalizable irrespective of the values $\left(c_{1}, c_{2}, c_{3}\right)$. This is presumably no longer the case for two or more loops, where more complicated terms such as $\kappa^{2} \operatorname{tr}\left(R_{\mu \nu \alpha \beta}{ }^{3}\right)$ may appear.

Returning to the Maxwell-Einstein case, we may use the field equations (2.4), (2.5), and (4.7) to reduce $(4.6)$ to the form

$$
\begin{aligned}
\Delta \mathscr{L}=(1 / \epsilon)(-g)^{1 / 2} & {\left[\alpha_{1} R_{\mu \nu}{ }^{2}+c_{5}\left(F^{2}\right)^{2}\right.} \\
& \left.+c_{6} R_{\mu \nu \alpha \beta} F^{\mu \nu} F^{\alpha \beta}\right] .
\end{aligned}
$$

Explicit calculation shows that $c_{5}=c_{6}=0$, but (unfortunately) $\alpha_{1} \neq 0$, as we now demonstrate. The nonghost matrices of $\mathrm{Eq}$. (4.1) have the form

$$
\begin{aligned}
& Y_{\mu \nu}=\left(\begin{array}{cc}
\partial_{\mu} \mathfrak{R}_{\nu}-\partial_{\nu} \mathfrak{R}_{\mu}-\frac{1}{2} Q_{\mu} Q_{\nu}+\frac{1}{2} Q_{\nu} Q_{\mu} & \partial_{\mu} Q_{\nu}-\partial_{\nu} Q_{\mu} \\
-\frac{1}{2} \partial_{\mu} Q_{\nu}+\frac{1}{2} \partial_{\nu} Q_{\mu} & \partial_{\mu} n_{\nu}-\partial_{\nu} n_{\mu}-\frac{1}{2} Q_{\mu} Q_{\nu}+\frac{1}{2} Q_{\nu} Q_{\mu}
\end{array}\right), \\
& X=\left(\begin{array}{cc}
2 X_{g}+P^{-1} X_{e}+\frac{1}{2} Q_{\mu} Q^{\mu}-\frac{1}{6} R & -\partial_{\mu} Q^{\mu} \\
-\frac{1}{2} \partial_{\mu} Q^{\mu} & R^{\alpha \beta}+\frac{1}{2} Q_{\mu} Q^{\mu}-\frac{1}{6} R
\end{array}\right),
\end{aligned}
$$

where we have used $P^{-1} Q=2 Q, P^{-1} X_{g}=2 X_{g}$. The traces of the diagonal and off-diagonal parts in $Y^{2}$ are given by ${ }^{14}$

$$
\begin{aligned}
& {\left[\left(\partial_{\mu} \Re_{\nu}-\partial_{\nu} \Re_{\mu}\right)-\frac{1}{2}\left(Q^{\mu} Q^{\nu}-Q^{\nu} Q^{\mu}\right)\right]^{2}=-24 R_{\mu \nu}^{2}+6 R^{2}-R F^{2}+3 F^{4}-\frac{9}{4}\left(F^{2}\right)^{2},} \\
& {\left[\left(\partial_{\mu} n_{\nu}-\partial_{\nu} n_{\mu}\right)-\frac{1}{2}\left(Q^{\mu} Q^{\nu}-Q^{\nu} Q^{\mu}\right)\right]^{2}=-4 R_{\mu \nu}{ }^{2}+R^{2}-2 R_{\alpha \beta} F_{2}^{\alpha \beta}+\frac{11}{2} F^{4}-\frac{13}{4}\left(F^{2}\right)^{2},} \\
& -2\left(\partial_{\mu} Q^{\nu}-\partial_{\nu} Q^{\mu}\right)^{2}=-\frac{9}{2}\left(D_{\alpha} F_{B \gamma}\right)^{2}+\left(D^{\alpha} F_{\alpha \beta}\right)^{2},
\end{aligned}
$$

Similarly, the $X^{2}$ terms are given by

$$
\begin{aligned}
& {\left[\left(2 X_{g}-\frac{1}{6} R\right)+P^{-1} X_{e}+\frac{1}{2} Q^{\mu} Q^{\mu}\right]^{2}=6 R_{\mu \nu}{ }^{2}+\frac{10}{36} R^{2}+\frac{1}{2} F^{4}-\frac{1}{8}\left(F^{2}\right)^{2},} \\
& \left(R^{\alpha \beta}-\frac{1}{6} R+\frac{1}{2} Q^{\mu} Q^{\mu}\right)^{2}=R_{\alpha \beta}{ }^{2}-\frac{2}{9} R^{2}+R_{\alpha \beta} F_{2}^{\alpha \beta}+\frac{1}{4} F^{4}+\frac{1}{2}\left(F^{2}\right)^{2}, \\
& \left(\partial_{\mu} Q^{\mu}\right)^{2}=\frac{3}{4}\left(D_{\alpha} F_{\beta \gamma}\right)^{2}+\frac{1}{2}\left(D^{\alpha} F_{\alpha \beta}\right)^{2},
\end{aligned}
$$

and the resulting nonghost counterterm is

$$
\Delta \mathscr{L}_{\mathrm{NG}}=(1 / \epsilon)(-g)^{1 / 2}\left[\frac{84}{120} R_{\mu \nu}^{2}+\frac{32}{120} R^{2}+\frac{1}{6} R_{\mu \nu} T^{\mu \nu}+\frac{13}{24} T_{\mu \nu}^{2}+\frac{1}{6}\left(D_{\alpha} F^{\alpha \beta}\right)^{2}\right] .
$$


Similarly, the ghost matrices are

$$
\begin{aligned}
& Y_{\mu \nu}{ }^{2}=-4 R_{\mu \nu}{ }^{2}+R^{2}, \\
& X^{2}=R_{\mu \nu}{ }^{2}+\left(\frac{1}{3}+\frac{5}{36}\right) R^{2}
\end{aligned}
$$

leading to the ghost contribution

$$
\Delta \mathscr{L}_{g}=-(1 / \epsilon)(-g)^{1 / 2}\left(\frac{1}{4} R_{\mu \nu}^{2}+\frac{7}{24} R^{2}\right) .
$$

The total counter-Lagrangian is the sum of (4.13) and (4.15), namely,

$$
\begin{aligned}
\Delta \mathscr{L}_{\text {tot }}=(1 / \epsilon)(-g)^{1 / 2} & {\left[\frac{9}{20} R_{\mu \nu}{ }^{2}-\frac{1}{40} R^{2}+\frac{1}{6} R_{\mu \nu} T^{\mu \nu}\right.} \\
& \left.+\frac{13}{24} T_{\mu \nu}{ }^{2}+\frac{1}{6}\left(D^{\alpha} F_{\alpha \beta}\right)^{2}\right] .
\end{aligned}
$$

Its dependence on the Maxwell field is only through the combination $T_{\mu \nu}$. Upon inserting the field equations, there remains

$$
\Delta \mathscr{L}_{\text {tot }}=(1 / \epsilon)\left(\frac{137}{60}\right)(-g)^{1 / 2} R_{\mu \nu} R^{\mu \nu} .
$$

This is not renormalizable, since we have already used the field equations as far as possible. ${ }^{12}$

We have no rigorous explanation of the fact that $\Delta \mathscr{L}$ depends on $F_{\mu \nu}$ only through the Maxwell stress tensor $T_{\mu \nu}$. It may be due to the identical tracelessness of the latter, or to the invariance of the classical Einstein-Maxwell field equations under constant duality rotations ${ }^{15,}{ }^{16} F_{\mu \nu}-F_{\mu \nu} \cos \alpha$ ${ }^{\star} F_{\mu \nu} \sin \alpha$, where ${ }^{\star} F_{\mu \nu} \equiv \frac{1}{2}(-g)^{-1 / 2} \epsilon^{\mu \nu \alpha \beta} F_{\alpha \beta}$ is the dual tensor. The stress tensor $T^{\mu \nu}=F_{2}^{\mu \nu}$ $+{ }^{\star} F_{2}^{\mu \nu}$ is manifestly invariant, but not the Maxwell Lagrangian, let alone $F_{\mu \nu} F_{\alpha \beta}$. The observed cancellations in the counter-Lagrangian for the Maxwell-Einstein system would thereby be accounted for to the extent that the symmetry of the classical field equations holds for the $S$ matrix as well. For massless scalars the situation is degenerate, since $\partial_{\mu} \phi \partial_{\nu} \phi$ is equivalent to $T_{\mu \nu}$, while invariance of the action under $\phi \rightarrow \phi+$ const guarantees that only $\partial \phi$ appears. For massless spinors, as we shall show elsewhere, there are non- $T_{\mu \nu}$ counterterms, at least for minimal coupling in the second-order formalism.

\section{DIVERGENCES OF OTHER GRAVITATIONAL MODELS}

It has been part of the folklore for many years that, as first suggested by Heisenberg, theories with dimensional coupling constants are nonrenormalizable because they have more singular high-energy behavior. In general relativity, the symptom is that the stress tensor to which the metric couples behaves as $p$ or $p^{2}$ (for fermions or bosons), so that attaching higher numbers of external gravitons to a loop does not lower its degree of divergence, while higher loops behave more and more singularly. Even with dimensional regularization, where everything is reduced to logarithmic divergences, we have seen that renormalizability is lost. One may therefore look for variants of Einstein theory which avoid dimensional constants. The Brans-Dicke (BD) theory ${ }^{17}$ was in fact motivated in part by the desire to replace $\kappa^{-1}$ by a field variable $\phi$, whose average value would provide a "Machian" basis for the strength of gravitation as a function of the matter content of the universe. It is therefore of interest to investigate whether this model is indeed an improvement over Einstein theory. ${ }^{18}$

The BD action is, in the absence of sources,

$$
\begin{aligned}
I_{\mathrm{BD}} & =\int d x \mathfrak{L}_{\mathrm{BD}} \\
& =-\int d x(-g)^{1 / 2}\left[\phi^{2} R+4 \omega \phi, \alpha \phi, \beta g^{\alpha \beta}\right],
\end{aligned}
$$

where $\phi$ is a scalar field of normal dimension $\left(L^{-1}\right)$ and $\omega$ a dimensionless parameter characterizing the scalar-tensor coupling (and $\omega \rightarrow \infty$ leads to ordinary Einstein theory). Now transform $\phi^{2} g_{\mu \nu} \equiv \bar{g}_{\mu \nu}$ to absorb the $\phi^{2}$ in the curvature scalar density. Using standard formulas for the scaling properties of a curvature, one finds (dropping the tilde)

$$
\begin{aligned}
I_{\mathrm{BD}}=- & \int d x(-g)^{1 / 2} \\
& \times\left[R+2(2 \omega+3)(\ln \phi)_{, \alpha}(\ln \phi)_{, \beta} g^{\alpha \beta}\right] .
\end{aligned}
$$

The choice of a new variable $\psi=[4(2 \omega+3)]^{1 / 2} \ln \phi$ then clearly reduces $(5.2 \mathrm{a})$ to the standard coupled scalar-Einstein for ${ }^{19}$

$$
I_{\mathrm{BD}}=-\int d x(-g)^{1 / 2}\left[R+\frac{1}{2} \psi_{, \alpha} \psi_{, \mathrm{B}} g^{\alpha \beta}\right] .
$$

This system, however, was already shown to be nonrenormalizable by ' $t$ Hooft and Veltman ${ }^{5}$ so that free Brans-Dicke theory is even worse than free Einstein theory. The $\omega=-\frac{3}{2}$ case is in general different from Einstein theory in the presence of matter (because the matter action becomes $\phi$-dependent), but the coupling to Maxwell theory remains unaltered by scaling and the negative conclusions of Sec. IV then apply. Although the above results are disappointing, it is conceivable that a deeper treatment of the $\phi$ field along the lines of a Higgs scalar might help. The gravitational constant corresponds, in Brans-Dicke theory, to some constant value $\phi_{0}$ of $\phi$. If this constant is in fact dictated by equilibrium considerations when matter is included, then perhaps a quantum expansion about that point would lead to improvement. We have not investigated this possibility further, but mention that the usual trick of adding terms $\sim(-g)^{1 / 2}\left(m^{2} \phi^{2}-\alpha \phi^{4}\right)$ to obtain nonzero $\phi_{0}$ in the absence of matter would 
lead to a transformed form $\sim(-g)^{1 / 2}\left(m^{2} e^{-a \psi}-\alpha\right)$ in the new variable. Also, in contrast with the vector case, one would be starting from a nonrenormalizable model here, and would hope it improves upon shifting.

An alternative way of avoiding a dimensional coupling constant is suggested by $\Delta \mathscr{L}$ itself. An initial action bilinear in the curvature such as the Weyl theory, with $I \sim \int d x\left(a R_{\mu \nu}{ }^{2}+b R^{2}\right)$ has two dimensionless parameters $(a, b)$. The resulting $\Delta \mathscr{L}$ would reproduce this form, but not involve $R^{3}$ or $R$ terms, but dimensions. Thus renormalization of $(a, b)$ would suffice. The classical Weyl theory suffers from great physical difficulties, but a variant of it has been revived recently. ${ }^{20}$ From the quantum point of view, the main problem is that the free graviton part of $R_{\mu \nu}{ }^{2}$ or $R^{2}$ is $\sim\left(\square h_{\mu \nu}\right)^{2}$ or $(\square h)^{2}$, and so has propagators behaving as $p^{-4}$ with the attendant ghost problems of higherorder derivative models. However, in view of its apparently renormalizable one-loop behavior, this model may repay closer investigation. As it stands, the Weyl theory is best suited to massless systems; in coupling to massive fields, one might pick up contributions to $\Delta \mathscr{L} \sim m^{2} R$, which would be unrenormalizable. In this connection, we may note that an initial action involving both $\kappa^{-2} R$ and a finite polynomial in $R^{2}$ would not help: for example, if $\mathscr{L} \sim \kappa^{-2} R+R^{2}, \Delta \mathcal{L}$ terms proportional to $\kappa^{2} R^{3}$ are to be expected (in addition to acceptable quadratic ones), which would spoil renormalizability. ${ }^{21}$ Thus the possibility that addition to the Einstein Lagrangian of only a finite number of counterterms would lead to a renormalizable theory appears highly unlikely because there would have to be a cancellation between (for example) the $\kappa^{2} R^{3}$ terms from the one- and two-loop diagrams.

A less drastic extension of classical general relativity is the addition of a cosmological term to the Einstein action $\sim \lambda \kappa^{-4}(-g)^{1 / 2}$, where $\lambda$ is dimensionless. There is now a dimensional constant even without sources. This system can be treated formally by the algorithm, but it is not very physical, for two reasons. First there is no weak field solution $g_{\mu \nu} \sim \eta_{\mu \nu}$; second the "mass" te rm from the expansion of $(-g)^{1 / 2}$ in Eq. (2.2b) has the form $\left(h_{\mu \nu}{ }^{2}-\frac{1}{2} h^{2}\right)$. This is well known to imply a ghost-scalar admixture; only the FierzPauli term $\left(h_{\mu \nu}{ }^{2}-h^{2}\right)$ corresponds to a ghost-free pure massive spin field. If one proceeds nevertheless, the only difference from the free Einstein field is the addition of the "mass term" $\sim \lambda \kappa^{-2}\left(\delta_{\mu \alpha} \delta_{\nu_{B}}\right.$ $\left.+\delta_{\mu \beta} \delta_{\nu \alpha}-\delta_{\alpha \beta} \delta_{\mu \nu}\right) \sim \lambda \kappa^{-2} P^{-1}$ to $X_{g}$ in (2.6). This in turn leads to an additional contribution,

$$
\Delta \mathscr{L}_{c} \sim(1 / \epsilon)(-g)^{1 / 2} \lambda \kappa^{-2}\left(\frac{\mathfrak{3}}{2} \lambda \kappa^{-2}-\frac{13}{12} R\right) .
$$

Since the field equations are now $R_{\mu \nu}=\frac{1}{2} \lambda \kappa^{-2} g_{\mu \nu}$, the total counterterm may be written in the equivalent forms

$$
\begin{aligned}
\Delta \mathcal{L} & =(1 / \epsilon)(-g)^{1 / 2} \lambda \kappa^{-4}\left(\frac{43}{60}\right) \\
& =(1 / \epsilon)(-g)^{1 / 2} \kappa^{-2} R\left(\frac{43}{120}\right),
\end{aligned}
$$

which can be absorbed in either the usual type of coupling-constant renor malization (here cosmological constant or $\kappa$ renormalization) or by a wave-function renormalization $g_{\mu \nu} \rightarrow g_{\mu \nu}+(a / \epsilon) g_{\mu \nu}$.

The free-field renormalizability is, of course, clear without calculation, since again the only invariants allowed are $(-g)^{1 / 2}\left(\kappa^{-4}, \kappa^{-2} R, R_{\mu \nu}{ }^{2}, R^{2}\right)$, all of which are harmless. However, the cosmological term does not improve matters in the presence of sources.

\section{CONCLUSIONS}

Already at the one-loop level, quantized general relativity is nonrenormalizable when coupled to scalars or photons. If one believes that gravitation must be quantized like all other fundamental fields, the nonrenormalizability of general relativity is a dilemma. Several possibilities present themselves. (a) Photons are not "fundamental" fields. Presumably fermions are still fundamental, and the crucial test is then whether this system is renormalizable. We will present our results on this in the accompanying paper, but merely state now that renormalizability is absent there, too. Also, the form of the contributions from various sources, scalar, vector, spinor, makes it unlikely that cancellations will occur for some particular mixture (see the Appendix). (b) Perturbative renormalizability is not the correct criterion for general relativity. Little can be said about this in our present state of knowledge, although it is certainly true that the classical self-energy of a point particle coupled to gravitation is divergent in a perturbation expansion in $\kappa$, but finite when the series is summed. ${ }^{22}$ (c) General relativity must be somehow "improved" at high frequencies through change of its basic Lagrangian or addition of appropriate nonminimal couplings to matter. We have briefly discussed some possible candidates in Sec. V, but this question is entirely open at present. It will be important, in investigating new models, to include coupling with matter, since we have seen that the source-free field can be misleadingly docile. Finally, there is the interesting, but technically difficult, problem of higherloop effects. Does free Einstein theory remain renormalizable, or do terms such as $\kappa^{2} \operatorname{tr}\left(R_{\mu \nu \alpha \beta}{ }^{3}\right)$ occur? 


\section{ACKNOWLEDGMENT}

We acknowledge several interesting discussions with H.-S. Tsao.

\section{APPENDIX}

It is of interest to isolate from the various divergent loop contributions the vacuum polarizations involving two vertices and pure graviton or pure matter internal lines. In the case of internal photons and fermions, for example, we can use scale invariance directly to determine the form of the counterterm contributions, while for any such loop we can also establish the signs of the counterterms by the usual positivity arguments for $\left\langle T_{\mu \nu} T_{\alpha \beta}\right\rangle$ expectations. Consider the photon loop, for example. It is described by the Maxwell action with quantized photons and background metric; this action being scale-invariant under the local transformation $g_{\mu \nu}(x) \rightarrow \lambda(x) g_{\mu \nu}(x)$, so is its contribution to $\Delta \mathscr{L}^{\gamma}$. But it is well known that the only curvature combination obeying scale invariance is the Weyl or conformal tensor

$$
\begin{aligned}
C_{\nu \alpha \beta}^{\mu}= & R_{\nu \alpha \beta}^{\mu} \\
& +\frac{1}{2}\left(\delta_{\alpha}^{\mu} R_{\nu \beta}+g_{\nu \beta} R_{\alpha}^{\mu}-g_{\nu \alpha} R_{\beta}^{\mu}-\delta_{\beta}^{\mu} R_{\nu \alpha}\right) \\
& +\frac{1}{6}\left(\delta_{B}^{\mu} g_{\nu \alpha}-\delta_{\alpha \delta_{\nu \beta}}^{\mu}\right) R .
\end{aligned}
$$

Consequently, $\Delta \mathscr{L}$ is necessarily proportional to the scale-invariant combination

$$
I_{2}=\frac{1}{2} \int d x C_{\nu \alpha \beta}^{\mu} C_{\mu \rho \sigma}^{\nu} g^{\alpha \rho} g^{B \sigma}(-g)^{1 / 2} .
$$

But (A1) together with the identity (4.7) implies

$$
I_{2}=\int d x(-g)^{1 / 2}\left(R_{\mu \nu} R^{\mu \nu}-\frac{1}{3} R^{2}\right) .
$$

To obtain the coefficient of $(1 / \epsilon) I_{2}$ one may either apply the algorithm to the photon part of $\mathscr{L}_{2}$ or compute the corresponding diagram with external gravitons at each vertex. The result is

$$
\int d x \Delta \mathscr{E}^{\gamma}=(1 / \epsilon)\left(\frac{1}{10}\right) I_{2} .
$$

The scale invariance of the Maxwell field is shared by that of a massless fermion field [but not by a (massless) scalar or tensor field], where $T_{\alpha}^{\alpha}=0$ by virtue of the Dirac equation. An exercise in Feynman integration then yields for the fermion loop

$$
\int d x \Delta \mathscr{L}^{F}=(1 / \epsilon)\left(\frac{1}{40}\right) I_{2} .
$$

We list also the contributions of $R^{2}$ type from the pure graviton loop, which are not scale-invariant ${ }^{23}$ :

$$
\int d x \Delta \mathcal{L}^{E}=(1 / \epsilon)\left(\frac{7}{20} I_{2}+\frac{5}{14} I_{0}\right),
$$

where $I_{0}=\int d x(-g)^{1 / 2} R^{2}$, and from the pure scalar loop:

$$
\int d x \Delta \mathcal{L}^{S}=(1 / \epsilon)\left(\frac{1}{120} I_{2}+\frac{1}{144} I_{0}\right)
$$

There are two features common to this list of spin $\left(0, \frac{1}{2}, 1,2\right)$ loops: The coefficient of the pure spin-two part $I_{2}$ is positive, while that of the pure scalar $I_{0}$ is non-negative. These results follow from unitarity or positivity considerations, and suggest that one should not expect renormalizability from some magic number of fields of appropriate spins. [Note, however, that cross terms like $R_{\mu \nu} T^{\mu \nu}$ can, and do, in Eq. (4.16a) have opposite on-shell contributions from $R_{\mu \nu}{ }^{2}$ and $T_{\mu \nu}{ }^{2}$.] One simple way to show positivity is to note that the vacuum polarization $\Pi_{\alpha \beta \mu \nu}=\left\langle 0\left|T_{\alpha \beta}(x) T_{\mu \nu}(y)\right| 0\right\rangle$, with conserved $T_{\mu \nu}$, is a positive sum of spin-2 and spin-0 intermediate states (conservation eliminates spin 1 and the other spin 0 ). This result holds as long as only physical intermediate particles are present, which is the case in the covariant quantization scheme when ghost contributions are included when needed. Thus, $\Pi_{\alpha \beta \mu \nu}$ is a sum of positive spectral contributions over tensors and scalars, and the required $\int \Delta \mathscr{L}=\int \phi_{\alpha \beta}$ $\times \Pi_{\alpha \beta \rho \sigma} \phi_{\rho \sigma}$ is easily seen to have the form ${ }^{24}$

$$
\int d x \Delta \mathscr{L}=\alpha I_{2}+\beta I_{0}, \quad \alpha, \beta \geqslant 0 .
$$

Clearly, when $T_{\alpha}^{\alpha}=0$, there is no spin-0 part, and $\beta=0$. There is no interesting case for which $\alpha$ vanishes, since this would result from a $T_{\mu \nu}$ having the form $\mathrm{m}^{25}\left(\square \eta_{\mu \nu}-\partial_{\mu} \partial_{\nu}\right) t$.
*Research supported by U. S. Air Force Office of Scientific Research, the National Science Foundation, and the Netherlands Ministerie van Onderwijs en Wetenschappen.

${ }^{1}$ R. P. Feynman, Acta Phys. Polon. 24, 697 (1963).

${ }^{2}$ B. S. DeWitt, Phys. Rev. 162, 1195 (1967); 162, 1239 (1967); and in Relativity, Groups and Topology (Gordon and Breach, London, 1964).
${ }^{3}$ L. D. Faddeev and V. N. Popov, Phys. Lett. 25B, 29 (1967); Kiev I.T.P. report (unpublished).

${ }^{4}$ Indeed, it has even been suggested that the gravitational field be exempt from quantization altogether, being perhaps some macroscopic average of a deeper underlying dynamics or a residue of better-behaved interactions.

${ }^{5}$ G. 't Hooft and M. Veltman, Ann. Inst. H. Poincaré (to 
be published); G. 't Hooft, Nucl. Phys. B62, 444 (1973).

${ }^{6} \mathrm{G}$. 't Hooft and M. Veltman, Nucl. Phys. B44, 189 (1972);

C. G. Bollini and J. J. Giambiagi, Phys. Lett. 40B, 566

(1972); Nuovo Cimento 12B, 189 (1972).

${ }^{7}$ One could have chosen some other function of the metric, such as $\bar{g}^{\mu \nu}$ or $(-\bar{g})^{1 / 2} \bar{g}^{\mu \nu}$, etc., to define the expansion, without altering the conclusions.

${ }^{8} \mathrm{~A}$ remark of $\mathrm{K}$. Johnson led us to this choice.

${ }^{9}$ It is perhaps useful to make two remarks concerning $\mathscr{L}_{2}$ within this framework. First, as to gauge: $\mathscr{L}_{2}$ alone is not invariant under (2.10); there are both inhomogeneous and homogeneous transformation contributions left over. The former, $\sim G^{\mu \nu}\left(h_{\mu \alpha, \nu}+h_{\nu \alpha, \mu}\right.$ $\left.-h_{\mu \nu}, \alpha\right) \eta^{\alpha}$, are canceled by homogeneous parts of $\mathscr{L}_{1}$ (and vanish at $G^{\mu \nu}=0$ anyway), while the latter are canceled by inhomogeneous parts from $\mathscr{L}_{3}$. But that is no problem, any more than the fact that in scalar electrodynamics only the sum of ordinary vertex $(e)$ plus seagull $\left(e^{2}\right)$ terms is gauge-invariant. Second, the form of $\mathfrak{L}_{2}$ will in general be different with different gauge choices, since the quadratic parts of various gauge-breaking terms will in general not be identical. Nor will the ensuing ghost contributions be identical, but the total $S$ matrix is gauge-invariant.

${ }^{10}$ The finite corrections are, of course, not so restricted, because they may have additional dependence on the external momenta, which supply further dimensions.

${ }^{11}$ R. Bach, Math. Z. 9 , 110 (1921); C. Lanczos, Ann. Math. 39, 842 (1938).

${ }^{12}$ Note that neither coupling-constant nor the usual wavefunction renormalization are of any use here. The former would only absorb counterterms proportional to $(1 / \epsilon) R$ which are absent; the latter $(g \rightarrow Z g)$ would again lead to the (rescaled) Einstein form.

${ }^{13}$ In two dimensions, there is a linear identity $g^{-1 / 2} \epsilon^{a b} \epsilon^{c d} R_{a b c d}=-2 g^{1 / 2} R=$ divergence. Curiously, these divergences depend explicitly on vierbein components rather than on the metric alone.

${ }^{14}$ The reader should be warned that care is required in tracing over the implicit indices, since there are only ten independent $h$ fields. For example, the nonghost contribution to the last term in Eq. (4.1) is $(10+4)$ $\times \frac{1}{60}\left(R_{\mu \nu}{ }^{2}-\frac{1}{3} R^{2}\right)$.

${ }^{15}$ G. Y. Rainich, Trans. Am. Math. Soc. 27, 106 (1925).
${ }^{16} \mathrm{C}$. W. Misner and J. A. Wheeler, Ann. Phys. (N.Y.) 2 , 525 (1957).

${ }^{17}$ C. Brans and R. H. Dicke, Phys. Rev. 124, 925 (1961).

${ }^{18}$ This possibility has also been suggested by S. Weinberg (private communication).

${ }^{19}$ Although the transformations involve $\varphi$ and therefore dimensions $\left(\hat{g}_{\mu \nu} \sim L^{-2}, \psi \sim L^{0}\right)$, one may write in expanding (6.2b) $\bar{g}_{\mu \nu}=\lambda^{-1}\left(g_{\mu \nu}+\lambda h_{\mu \nu}\right), \bar{\psi}=\psi+\lambda \varphi$ to restore correct dimensions to the background and quantum fields. At this level, $\lambda$ has dimensions of $\kappa$, but is otherwise arbitrary.

${ }^{20}$ For a discussion of this model, see S. Deser, Ann. Phys. (N.Y.) 59, 248 (1970); P. A. M. Dirac, Proc. R. Soc. A333, 403 (1973).

${ }^{21}$ This expectation is based on treating the $R^{2}$ term in the usual perturbation sense as a new vertex rather than as the leading $\left(p^{-4}\right)$ contribution to the propagator (see also Ref. 2). A "constructive" approach to an improved theory might be to add ad hoc terms to $\mathscr{L}_{2}$ of the form $A^{\mu \nu \alpha \beta} h_{\mu \alpha} h_{\nu \beta}$ with coefficients $A$ (if possible) such that the resulting $\Delta \mathscr{L}$ vanishes. However, this scheme would only be consistent if there existed a gauge-preserving (i.e., generally covariant) $\mathcal{L}^{\prime}(\bar{g})$ which provided those terms, without altering the observed lowfrequency properties of Einstein's equations (i.e., without disturbing $\mathscr{L}_{1}$ too much), and without introducing ghosts. See, in this connection, D. G. Boulware and S. Deser (to be published).

${ }^{22}$ R. Arnowitt, S. Deser, and C. W. Misner, Ann. Phys. (N.Y.) $\underline{33}, 88$ (1965).

${ }^{23}$ The term (A4) agrees with the calculation of D. M. Capper, M. J. Duff, and L. Halpern, this issue, Phys. Rev. D 10, 461 (1974); (A5) coincides with recently corrected calculations of Ref. 2 (private communication) and D. M. Capper and M. J. Duff, [ICTP Report No. 73/12 (unpublished)], while (A6) was first calculated in Ref. 5, and (A7) was obtained in Refs. 1 and 2.

${ }^{24}$ Contract the spin-2, -0 projection operators in II with weak external fields $\varphi_{\mu \nu}=g_{\mu \nu}-\eta_{\mu \nu}$ to obtain the forms $\frac{1}{4}\left[\left(\square \varphi_{\mu \nu}\right)^{2}-\frac{1}{3}(\square \varphi)^{2}\right] \sim I_{2}$ and $\frac{1}{4}(\square \varphi)^{2} \sim I_{0}$.

${ }^{25}$ The "new improved" part of the scalar field's stress tensor is of this type (with $t=\frac{1}{6} R^{2}$ ), being in fact so constructed as to make $T_{\alpha}^{\alpha}=0$. 